\title{
Modelos para determinação dos parâmetros da equação de van Genuchten para um Cambissolo
}

\author{
Carlos R. de Mello', Geraldo C. de Oliveira ${ }^{2}$, Daniel F. Ferreira ${ }^{3}$, José M. de Lima ${ }^{4}$ \& Diego Lopes ${ }^{4}$ \\ 1 DEG/UFLA. Campus Universitário, CP 37, CEP 37200-000, Lavras, MG. Fone: (35)3821-7143. \\ E-mail: rog.mello@terra.com.br (Foto) \\ 2 Escola de Agronomia, Setor de Solos, UFG, CEP 74001-970, Goiânia, GO. E-mail: gcesar@agro.ufg.br \\ ${ }^{3}$ DEX/UFLA. Fone: (35)3929-1369. E-mail: dfferreira@ufla.br, bolsista do CNPq. \\ ${ }^{4}$ DCS/UFLA. Fone: (35)3829-1332. E-mail: jmlima@ufla.br, bolsista do CNPq; Lopessrs@yahoo.com.br, bolsista FAPEMIG.
}

Protocolo 98 - 2/6/2003 - Aprovado em 6/4/2004

\begin{abstract}
Resumo: Com este trabalho, objetiva-se a geração de modelos matemáticos para predição dos 4 parâmetros da equação de van Genuchten ( $\theta R, \theta S, \alpha$ e n) e para a umidade correspondente à capacidade de campo em função dos atributos areia, argila, matéria orgânica e densidade do solo, ambas de fácil e rotineira determinação em laboratório, além da profundidade. Coletaram-se 36 amostras não deformadas e deformadas nas profundidades de 0 a 30, 30 a 60 e 60 a $90 \mathrm{~cm}$, em Cambissolo originado de gnaisse, determinando-se as umidades correspondentes às tensões de 2, 6 e $10 \mathrm{kPa}$ em mesa de tensão e as referentes a 33, 100, 500 e $1500 \mathrm{kPa}$, em câmara de Richards. Para ajuste dos modelos, trabalhou-se com o programa SAS for Windows, com a rotina Proc Reg, e procedimento Backward, para selecionar as variáveis significativamente diferentes de zero num certo nível de probabilidade. Para avaliação dos modelos, consideraram-se o coeficiente de determinação e os erros de predição, tanto para os parâmetros estimados quanto para as umidades geradas pela equação de van Genuchten ajustada com os parâmetros estimados, para cada tensão descrita acima. Constatou-se que os erros foram consideravelmente pequenos, justificando a aplicação dos modelos.
\end{abstract}

Palavras-chave: irrigação, umidade do solo, curva característica

\section{Models for determination of parameters of van Genuchten equation for a Cambisol}

\begin{abstract}
This work proposes to adjust mathematical models to predict the parameters of van Genuchten equation ( $\theta \mathrm{R}, \theta \mathrm{S}, \alpha$ and $\mathrm{n}$ ), besides a model to predict the soil moisture corresponding to the field capacity. The models express the equation parameters as dependent variables and sand, clay, organic matter, bulk density and depth as independent variables. All independent variables can be easily and routinely determined in laboratory. The samples were collected at 12 points in and three layers $(0-30 ; 30-60 ; 60-90 \mathrm{~cm})$ of a Cambisol, using a Uhland sampler. The soil moisture at matric potentials of 2,6 and $10 \mathrm{kPa}$ were determined using a porous plate apparatus and those at matric potentials of 33,100,500 and $1500 \mathrm{kPa}$ were determined using a pressure plate apparatus. The models were adjusted with the SAS for Windows program, ProcReg routine and Backward procedure to select the variables significantly different from zero at the probability level adopted. The coefficient of determination and prediction errors for the parameters predicted and soil moisture generated by van Genuchten equation adjusted with parameters predicted by models, were analyzed to evaluate the models. The coefficient of determination for models was high and the errors were small justifying the application of models.
\end{abstract}

Key words: irrigation, soil moisture, water retention curve 


\section{INTRODUÇÃO}

O uso de modelos matemáticos para predição de atributos de difícil determinação na ciência do solo, em especial os físicohídricos, é uma boa alternativa devido ao pequeno banco de dados para as várias unidades pedológicas, dificuldades de determinação direta em laboratório, grande dispêndio de tempo e dinheiro. Existem atributos físico-hídricos do solo cuja determinação somente é finalizada em laboratório após duas ou mais semanas, estando condicionados a caraterísticas intrínsecas do solo, dentre as quais se destaca a determinação da curva característica do solo, que expressa a umidade em função do potencial matricial da água no solo (Libardi, 1995; Sales et al., 1999; Hodnett \& Tomasella, 2002 ).

De posse da curva característica de água no solo, são possíveis vastas aplicações práticas e científicas, destacandose a porosidade drenável (Mello et al., 2002a), capacidade de campo (Ferreira \& Marcos, 1983; Fabian \& Ottoni Filho , 2000; Mello et al., 2002b) disponibilidade total de água (Arruda et al., 1987; Centurion \& Andrioli, 2000; Mello et al., 2002a), e condutividade hidráulica não-saturada (van Genutchen, 1980; Wösten et al., 1990; Tomasella \& Hodnett, 1997), além do manejo de irrigação e balanço hídrico, determinando-se variações de armazenamento de água no solo.

A possibilidade de estimativa da curva característica com base em atributos de determinação rotineira, muitas vezes constantes em levantamentos pedológicos, como textura, matéria orgânica e densidade do solo, pode ser extremamente útil para avaliações técnico-financeiras de projetos de irrigação e drenagem, além de projetos para assentamento rural, classificação de solos e Levantamentos Conservacionistas, visando ao uso racional do recurso terra (Mello et al., 2002a; Brasil, 1983). Em tais situações é de suma importância a determinação rápida e precisa da curva característica, o que é possível através de modelagem matemática.

A relação entre propriedades hidráulicas do solo com atributos físicos vem sendo estudada com o intuito de se conhecer suas possíveis correlações, haja vista que já foi comprovada a influência dos teores de argila, areia e matéria orgânica na capacidade de retenção de água pelo solo. Vários pesquisadores, destacando-se Centurion \& Andrioli (2000), Zhuang et al. (2001), Hodnett \& Tomasella (2002) e Mello et al. (2002a), obtiveram correlações significativas entre algumas propriedades hidráulicas do solo com atributos físicos, em especial aqueles de determinação rotineira, como textura, matéria orgânica e densidade do solo.

Os Cambissolos são unidades pedológicas comuns na região Sul de Minas Gerais e Campos das Vertentes, explorados para cultura do café, citrus e pastagem, além de comporem grande parte do entorno dos reservatórios de usinas hidrelétricas, como Furnas, Camargos e Funil (Oliveira, 1993; Giarola et al., 1997). Devido à sua gênese, esses solos são altamente susceptíveis a problemas ambientais, como elevadas perdas de solo e contaminações, especialmente da água, tanto subterrânea quanto superficial. Esses problemas estão associados ao fato de que os Cambissolos são unidades pedológicas em formação, caracterizadas com horizonte B incipiente, normalmente com elevados teores de silte, sobretudo em profundidade. Estes, por sua vez, são componentes texturais pouco desejáveis no contexto conservacionista tropical, devido à sua capacidade de se desprender pelo impacto de gotas de chuva e/ou irrigação (salpicamento) e, produzir um selamento superficial, dificultando infiltração de água e aumento de escoamento superficial e erosão (Resende et al., 1999). Desta forma, é de substancial importância o manejo adequado desses solos, o que poderá ser facilitado pelo bom conhecimento de seus parâmetros hídricos, em sua maioria expressos pela curva característica (Silveira et al., 2001).

Ante o exposto objetiva-se, com este trabalho, o desenvolvimento de modelos matemáticos lineares para estimativa dos parâmetros da equação de van Genuchten, que é a mais usada para representar a curva característica do solo, com base nos atributos areia, argila, matéria orgânica e densidade do solo.

\section{MATERIAL E MÉTODOS}

Foram coletadas 36 amostras representativas nãodeformadas e deformadas de um Cambissolo originado de gnaisse, nas profundidades de 0 a 30, 30 a 60 e 60 a 90 cm, numa bacia hidrográfica próxima ao AHF (Aproveitamento Hidrelétrico do Funil) a cerca de $11 \mathrm{~km}$ do município de Lavras, MG, sendo 12 amostras por camada. As amostras não deformadas foram coletadas com auxílio de amostrador do tipo Uhland, saturadas, posteriormente levadas à mesa de tensão para obtenção das umidades correspondentes às tensões de 2,6 e $10 \mathrm{kPa}$, pelo processo de secagem, após estabilização. As amostras deformadas foram peneiradas, obtendo-se agregados menores que 2,0 mm, saturadas e levadas à Câmara de Richards, para estabilização e posterior determinação da umidade correspondente às tensões de 33, 100, 500 e $1500 \mathrm{kPa}$.

A análise textural do solo foi feita pelo método de Bouyoucos (Gee \& Bauder, 1986); a densidade do solo, pelo método do cilindro de Uhland (EMBRAPA, 1997) e carbono orgânico obtido da oxidação da matéria orgânica via úmida (EMBRAPA, 1997). A matéria orgânica foi obtida pela multiplicação do carbono orgânico por 1,724 (Walkley \& Black, 1934). A porosidade do solo foi determinada com base na umidade volumétrica de saturação do solo, a microporosidade correspondendo à umidade resultante quando a amostra é submetida à sucção de $6 \mathrm{kPa}$ e a macroposidade por diferença entre as anteriores (Dias Júnior et al., 2000). Na Tabela 1 constam os atributos físicos médios do solo, nas profundidades estudadas e o desvio padrão. Na seqüência apresentam-se, na Figura 1, as curvas características médias das camadas estudadas.

A seguir, ajustou-se a equação proposta por van Genuchten (1980) para obtenção dos parâmetros, a qual possui a seguinte fórmula matemática:

$$
\theta=\theta \mathrm{R}+(\theta \mathrm{S}-\theta \mathrm{R}) \cdot\left[\frac{1}{1+\left(\alpha \cdot \psi_{\mathrm{m}}\right)^{\mathrm{n}}}\right]^{\mathrm{m}}
$$


Tabela 1. Atributos físicos* do Cambissolo nas profundidades monitoradas

\begin{tabular}{|c|c|c|c|c|c|c|c|c|}
\hline \multirow{2}{*}{ Camada (cm) } & \multirow[t]{2}{*}{ Areia } & \multirow{2}{*}{$\begin{array}{l}\text { Argila } \\
\mathrm{g} \mathrm{kg}^{-1}\end{array}$} & \multirow[t]{2}{*}{ Silte } & \multicolumn{2}{|c|}{ Porosidade** } & \multirow{2}{*}{$\begin{array}{c}\text { DS } \\
\mathrm{kg} \mathrm{dm}^{-3}\end{array}$} & \multirow{2}{*}{ PT } & \multirow{2}{*}{$\begin{array}{c}\mathrm{Mo} \\
\mathrm{g} \mathrm{kg}^{-1}\end{array}$} \\
\hline & & & & Micro & Macro & & & \\
\hline $0-30$ & 503 & 239 & 258 & 0,361 & 0,138 & 1,50 & 0,499 & 15,7 \\
\hline $30-60$ & 469 & 237 & 294 & 0,334 & 0,142 & 1,52 & 0,476 & 09,3 \\
\hline $60-90$ & 423 & 208 & 369 & 0,321 & 0,134 & 1,52 & 0,455 & 07,6 \\
\hline Desvio & 122,5 & 89,2 & 126 & 0,042 & 0,025 & 0,106 & 0,055 & 04,72 \\
\hline
\end{tabular}

*PT - porosidade total; DS - densidade do solo; MO - materia orgânica; Desvio - desvio padrão das determinações.
** Valores $\mathrm{em} \mathrm{m}^{3} \mathrm{~m}^{-3}$

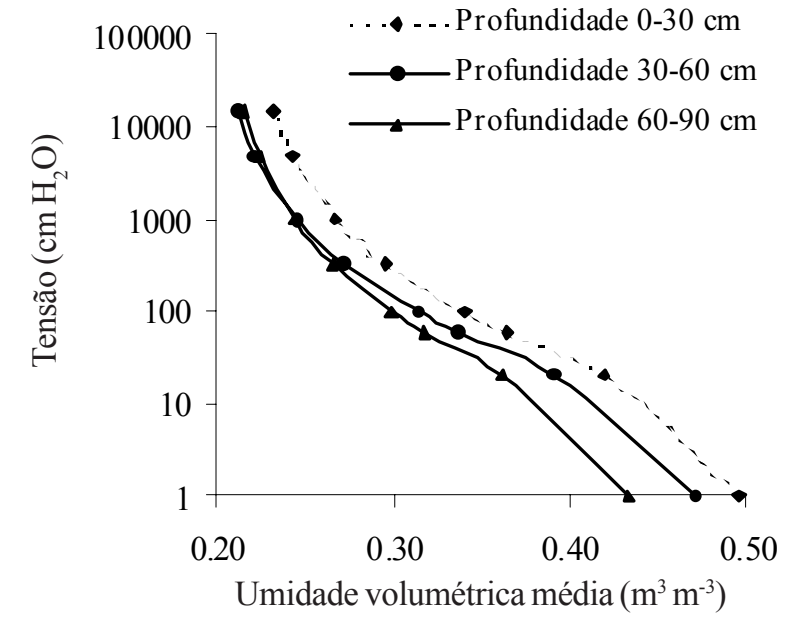

Figura 1. Curvas características médias para cada profundidade monitorada do Cambissolo na microbacia

com $\mathrm{m}=1-\frac{1}{\mathrm{n}}$ (Mualem, 1976), sendo $\theta$ a umidade volumétrica, $\psi_{\mathrm{m}}$ o potencial matricial; $\theta \mathrm{R}$ e $\theta \mathrm{S}$, respectivamente, a umidade volumétrica residual e na saturação e n e $\alpha$ parâmetros de ajuste. O parâmetro $\theta \mathrm{CC}$ (umidade na capacidade de campo) foi obtido considerando-se a umidade correspondente à inflexão da curva característica, conforme Ferreira \& Marcos (1983) e Mello et al. (2002b).

Desta forma, foram ajustados modelos matemáticos para estimativa dos parâmetros (1 para cada parâmetro) da equação de van Genutchen. Os parâmetros modelados foram alfa $(\alpha), n$, $\theta \mathrm{R}, \theta \mathrm{S}$ e $\theta \mathrm{CC}$, totalizando 5 modelos matemáticos; no seu ajuste trabalhou-se com o programa SAS for Windows, com a rotina ProcReg e procedimento Backward (SAS, 1985). A rotina ProcReg corresponde ao procedimento de regressão linear múltipla do programa SAS, o qual estima os coeficientes dos modelos de regressão por meio do método de mínimos quadrados. O procedimento Backward foi aplicado devido à sua característica de selecionar as variáveis significativamente diferentes de zero, de acordo com o nível de significância adotado, identificando e eliminando aquelas que não estão colaborando para a qualidade final do modelo. Para todos os parâmetros foi adotado nível de significância de $5 \%$, exceção feita ao parâmetro alfa $(\alpha)$, para o qual a inclusão de variáveis significativas a $10 \%$ produziu substancial melhora no coeficiente de determinação; tal procedimento foi adotado devido ao fato deste parâmetro apresentar maior dificuldade de ajuste, conforme constatado por outros trabalhos, em especial Tomasella et al. (2000) e Hodnett \& Tomasella (2002).

Inicialmente, foi estruturado um modelo único, constituído por variáveis de entrada geradas a partir da combinação entre aquelas individuais (areia, argila, matéria orgânica, densidade do solo e profundidade); no entanto, o modelo final para o respectivo parâmetro foi obtido a partir da seleção das variáveis significativas pelo procedimento Backward, proporcionando a produção de um modelo específico para o mesmo. A variável profundidade também foi considerada, haja vista as variabilidades entre as camadas, produzidas principalmente pelas características texturais, havendo aumento considerável de silte com a profundidade (Tabela 1) e este comportamento, típico de cambissolos, tem influência predominante nas propriedades hidráulicas do mesmo (Oliveira, 1993) e, neste caso, foram considerados os valores de 20,50 e $80 \mathrm{~cm}$, respectivamente, para as camadas de 0-30, 30-60 e 60-90, por se tratar de valores intermediários representativos da profundidade de coleta, levando-se em conta o amostrador Uhland e suas dimensões.

A análise da precisão do modelo foi feita considerando-se o coeficiente de determinação $\left(\mathrm{R}^{2}\right)$, as significâncias dos parâmetros estimados para as variáveis selecionadas pelo Backward, erros médio, máximo e mínimo, produzidos pelo respectivo modelo em relação aos valores originais, e por meio de análise da dispersão dos pontos em torno da reta 1:1, feita com base nas estimativas da umidade pela equação de van Genutchen gerada pelos parâmetros estimados pelos modelos propostos e pela equação de van Genutchen, com base nos dados de tensão e umidade obtidos em laboratório, produzindose um coeficiente de determinação para a reta de cada tensão testada. Os erros foram calculados através da seguinte equação:

$$
\mathrm{e}(\%)=\frac{\left|\mathrm{V}_{\text {original }}-\mathrm{V}_{\text {estimado }}\right|}{\mathrm{V}_{\text {original }}} \cdot 100
$$

em que $\mathrm{V}_{\text {original }}$ é o valor original do parâmetro $\mathrm{V}_{\text {estimado }}$, o valor estimado do mesmo.

\section{RESULTADOS E DISCUSSÃO}

Na Tabela 2 apresentam-se a média, o desvio padrão e o coeficiente de variação dos parâmetros da equação de van Genuchten e dos atributos físicos do solo utilizados no ajuste dos modelos. Ao analisá-la é possível verificar pequena variabilidade dos parâmetros da equação de van Genuchten, exceção feita ao parâmetro alfa $(\alpha)$, o qual possui elevado coeficiente de variação. Além disto, o atributo matéria orgânica também apresenta considerável coeficiente de variação, o que, possivelmente, foi produzido pelos maiores teores na camada superior em relação às inferiores, conforme se pode observar na Tabela 1. Chama atenção também a elevada densidade do 
solo explicada pelas características do Cambissolo, com elevado percentual de partículas minerais grosseiras, e também ao uso intensivo do solo pelo pastejo de gado de corte há pelo menos 10 anos.

Tabela 2. Média, desvio padrão e coeficiente de variação dos parâmetros da equação de van Genutchen e atributos texturais utilizados no ajuste dos modelos*

\begin{tabular}{crcr}
\hline Parâmetro & Média & Desvio & CV \\
\hline$\alpha$ & 0,12966 & 0,064 & 49,6 \\
$\mathrm{n}$ & 1,35301 & 0,077 & 5,7 \\
$\theta \mathrm{R}$ & 0,196 & 0,040 & 20,6 \\
$\theta \mathrm{S}$ & 0,477 & 0,055 & 11,5 \\
$\theta \mathrm{CC}$ & 0,382 & 0,051 & 13,5 \\
Areia & 46,50 & 12,25 & 25,0 \\
Argila & 22,80 & 8,919 & 31,1 \\
MO & 1,087 & 0,472 & 43,5 \\
DS & 1,52 & 0,106 & 7,0 \\
\hline * CV - Coeficiente de variação; MO - matéria orgânica: DS - densidade do solo
\end{tabular}

Todos os modelos foram ajustados com base nas variáveis oriundas dos percentuais dos atributos areia e argila e na profundidade. Nos modelos para os parâmetros $n$ e $\theta \mathrm{R}$, as variáveis baseadas na densidade do solo não foram significativas e nos modelos para os parâmetros $\theta \mathrm{S}$ e $\theta \mathrm{CC}$, as variáveis baseadas no percentual de matéria orgânica também não foram significativas. $\mathrm{O}$ atributo silte, por ser determinado por diferença entre o total textural e a soma de areia e argila, produziu covariância com essas últimas, e foi descartado. Os ajustes mostraram a significância com que os atributos texturais influenciam as propriedades hidráulicas do solo, na figura das curvas características, conforme constatado por Mello et al. (2002a) estudando a porosidade drenável e a disponibilidade total de água no solo. No entanto, os citados autores não encontraram significância para matéria orgânica, e Gupta \& Larson (1979) salientaram tal importância, o que também foi constatado por este trabalho para alguns parâmetros. Assim, para determinação da curva característica como um todo, é imprescindível a presença do percentual de matéria orgânica e da densidade do solo, além da textura. Para determinar a curva característica basta conhecer $\psi_{\mathrm{m}}$ versus $\theta$. É conveniente destacar também que a profundidade é uma variável a ser considerada nos modelos, mostrando haver variabilidade das características físico-hídricas em profundidade, o que pode também ser verificado na Figura 1 e na Tabela 1.

$\mathrm{Na}$ Tabela 3 (A, B, C, D e E) constam as estimativas dos coeficientes para as variáveis independentes dos modelos dos parâmetros da equação de van Genutchen, bem como significâncias estatísticas. Pode-se analisar a elevada significância dos parâmetros não apenas a nível de 5\%, mas a $1 \%$, para a maior parte das estimativas. As menores significâncias podem ser observadas para o parâmetro alfa $(\alpha), 0$ qual apresentou maior dificuldade de ajuste, o que pode ser demonstrado pelo elevado coeficiente de variação apresentado pelos dados, gerando a mais alta variabilidade, o que não foi seguido pelos atributos do solo (Tabela 2).

Na Tabela 4 apresentam-se os coeficientes de determinação e erros produzidos pelos modelos, os quais permitem avaliar a sua qualidade.
Tabela 3. Estimativa dos coeficientes das variáveis dos modelos ajustados para os parâmetros de van Genuchten $(\alpha, n, \theta \mathrm{R}$, $\theta \mathrm{S}, \theta \mathrm{CC})$ e significância estatística dos mesmos*

\begin{tabular}{|c|c|c|}
\hline Variável & Estimativa & Prob $>|t|$ \\
\hline \multicolumn{3}{|l|}{ A. $\alpha$} \\
\hline Intercepto & 5,6869 & $0,0001 * *$ \\
\hline Areia & $-0,06062$ & $0,0001 * *$ \\
\hline MO & $-1,28823$ & $0,0006 * *$ \\
\hline DS & $-4,8706$ & $0,0001 * *$ \\
\hline$(\arg )^{2}$ & $-7,785 \times 10^{-4}$ & $0,0016 * *$ \\
\hline$(\mathrm{MO})^{2}$ & 0,11259 & $0,0017 * *$ \\
\hline$(\text { prof })^{2}$ & $3,252 \times 10^{-5}$ & $0,0305^{*}$ \\
\hline areia MO & 0,00929 & $0,0015 * *$ \\
\hline areia DS & 0,05613 & $0,0001 * *$ \\
\hline $\arg$ DS & 0,05490 & $0,0010 * *$ \\
\hline arg prof & $-9,944 \times 10^{-5}$ & $0,0708 \dagger$ \\
\hline MO DS & 0,37632 & $0,0863 \dagger$ \\
\hline$(\text { areia })^{2} \arg$ & $-1,385 \times 10^{-5}$ & $0,0009^{* *}$ \\
\hline \multicolumn{3}{|l|}{ B. $n$} \\
\hline Intercepto & $-5,86738$ & $0,0016 * *$ \\
\hline Areia & 0,17432 & $0,0004 * *$ \\
\hline Arg & 0,17778 & $0,0014 * *$ \\
\hline MO & 0,51456 & $0,0042 * *$ \\
\hline Prof & 0,02440 & $0,0002 * *$ \\
\hline$(\text { areia })^{2}$ & $-0,00123$ & $0,0004 * *$ \\
\hline$(\arg )^{2}$ & $-9,675 \times 10^{-4}$ & $0,0162 *$ \\
\hline$(\mathrm{MO})^{2}$ & $-0,16995$ & $0,0011 * *$ \\
\hline areia arg & $-0,00339$ & $0,0010 * *$ \\
\hline areia prof & $-1,931 \times 10^{-4}$ & $0,005^{* *}$ \\
\hline$(\text { areia })^{\frac{2}{M O}}$ & $-1,062 \times 10^{-4}$ & $0,0076 * *$ \\
\hline$(\text { areia })^{2} \arg$ & $3,265 \times 10^{-5}$ & $0,0003 * *$ \\
\hline areia arg prof & $-1,283 \times 10^{-5}$ & $0,0003 * *$ \\
\hline \multicolumn{3}{|l|}{ C. $\theta \mathrm{R}$} \\
\hline Intercepto & 0,41774 & $0,0001 * *$ \\
\hline Areia & $-0,0048$ & $0,0001 * *$ \\
\hline MO & $-0,31567$ & $0,0001 * *$ \\
\hline$(\arg )^{2}$ & $6,617 \times 10^{-5}$ & $0,0053 * *$ \\
\hline$(\text { prof })^{2}$ & $1,025 \times 10^{-5}$ & $0,0082 * *$ \\
\hline areia $\mathrm{MO}$ & 0,01006 & $0,0001 * *$ \\
\hline arg prof & $-4,585 \times 10^{-5}$ & $0,0048 * *$ \\
\hline$(\text { areia })^{2} \mathrm{MO}$ & $-7,3 \times 10^{-5}$ & $0,0006^{* *}$ \\
\hline \multicolumn{3}{|l|}{ D. $\theta \mathrm{S}$} \\
\hline Intercepto & 0,75934 & $0,0001 * *$ \\
\hline$(\text { areia })^{2}$ & $-1,894 \times 10^{-5}$ & $0,0001 * *$ \\
\hline$(\mathrm{DS})^{2}$ & $-0,09296$ & $0,0001 * *$ \\
\hline areia arg prof & $-3,0253 \times 10^{-7}$ & $0,0288^{*}$ \\
\hline
\end{tabular}

\begin{tabular}{|c|c|c|}
\hline \multicolumn{3}{|l|}{ E. $\theta \mathrm{CC}$} \\
\hline intercepto & $-0,78706$ & $0,1982^{\mathrm{ns}}$ \\
\hline DS & 1,8630 & $0,0281 *$ \\
\hline$(\text { areia })^{2}$ & $-9,212 \times 10^{-5}$ & $0,0001 * *$ \\
\hline$(\arg )^{2}$ & $2,176 \times 10^{-4}$ & $0,0026^{* *}$ \\
\hline$(\mathrm{DS})^{2}$ & $-0,56118$ & $0,0357 *$ \\
\hline $\arg$ DS & $-0,01162$ & $0,0022 * *$ \\
\hline$(\text { areia })^{2} \arg$ & $3,05 \times 10^{-6}$ & $0,0002 * *$ \\
\hline Areia arg prof & $-2,3045 \times 10^{-7}$ & $0,0288^{*}$ \\
\hline
\end{tabular}
respectivamente, umidade residual, saturação e capacidade de campo.

O modelo para o parâmetro alfa $(\alpha)$ possui bom coeficiente de determinação e erro médio aceitável. No entanto, produziu um erro máximo muito elevado, o que o diferencia dos demais 
Tabela 4. Coeficientes estatísticos obtidos pelos modelos ajustados para os parâmetros da equação de van Genutchen

\begin{tabular}{ccccc}
\hline Modelo & $\mathrm{R}^{2}$ & Médio & Máximo & Mínimo \\
\hline$\alpha$ & 0,7594 & 26,68 & 180,21 & 0,034 \\
$\theta \mathrm{R}$ & 0,9058 & 5,11 & 12,75 & 0,168 \\
$\theta \mathrm{S}$ & 0,7834 & 4,37 & 12,87 & 0,238 \\
$\theta \mathrm{CC}$ & 0,8922 & 3,49 & 13,35 & 0,001 \\
$\mathrm{n}$ & 0,7234 & 2,17 & 8,48 & 0,117 \\
\hline
\end{tabular}

modelos. O modelo para o parâmetro n apresentou um $\mathrm{R}^{2}$ inferior ao do modelo para $\alpha$, mas apresentou erros muito inferiores, podendo-se caracterizá-lo como de melhor precisão. Tomasella et al. (2000) trabalhando com vários solos brasileiros geraram um coeficiente de determinação máximo igual a 0,4124, mesmo trabalhando com o parâmetro alfa transformado para forma logarítmica. Para n, os autores encontraram valor máximo de $\mathrm{R}^{2}$ igual a 0,3703 ; para $\theta \mathrm{S}$ um valor de $0,84 \mathrm{e}$, para $\theta \mathrm{R}$, 0,83 , concluindo sobre a boa qualidade de seus ajustes. É conveniente se destacar, também, que esses autores trabalharam com mais variáveis, considerando-se especialmente dados de textura fracionada. Destaca-se que os melhores ajustes ocorreram para os modelos de $\theta \mathrm{R}$ e $\theta \mathrm{CC}$, onde é possível observar que não há diferença entre os coeficientes de determinação dos mesmos e os erros produzidos foram consideravelmente baixos. Apesar de indicar um coeficiente de determinação menor em comparação com $\theta \mathrm{R}, \theta \mathrm{CC}$ e
Tomasella et al. (2000), o modelo para $\theta$ S possui boas qualidades estatísticas, uma vez que produziu erros baixos, na mesma magnitude dos modelos citados anteriormente e o número de variáveis utilizadas para ajuste do mesmo foi consideravelmente inferior, mostrando o bom ajuste desenvolvido. Assim, os resultados obtidos neste trabalho mostram a boa qualidade dos modelos, comprovada não só pelo coeficiente de determinação mas, também, pelos baixos erros proporcionados e pela comparação de suas qualidades estatísticas com as de outros modelos existentes.

$\mathrm{Na}$ Tabela 5 apresentam-se os erros obtidos pelos modelos quando comparadas as umidades geradas pela equação de van Genuchten original e a ajustada com os parâmetros estimados pelos modelos. É possível se observar a alta precisão dos modelos quando aplicados para determinação da umidade, o que se deve aos pequenos erros encontrados, inclusive aos máximos, permitindo a qualificação da modelagem proposta.

Ainda com relação a este aspecto, os gráficos da Figura 2 mostram o desempenho da equação de van Genutchen ajustada com base nas estimativas dos modelos dos parâmetros propostos por este trabalho, em comparação com os modelos originais de cada ponto e profundidade, com ambos gerando um valor de umidade para cada tensão.

Observa-se que, de maneira geral, há pequena dispersão dos pontos ao redor da reta 1:1, mostrando a boa aproximação entre os valores estimados e os originais. Destaca-se, também, aumento do coeficiente de determinação da reta $1: 1$ à medida
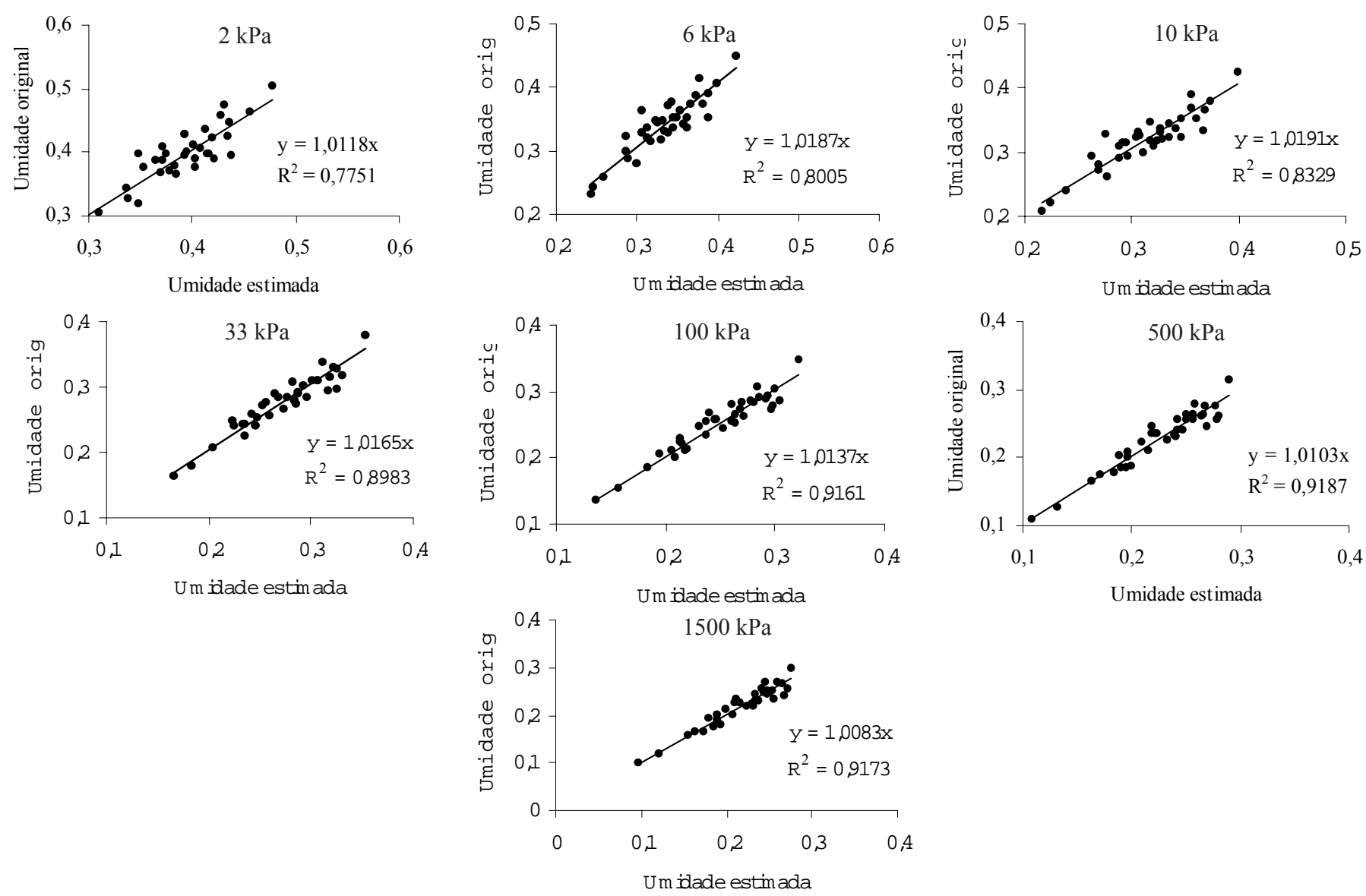

Figura 2. Dispersão dos valores estimados em torno da reta 1:1 a diferentes tensões. 
Tabela 5. Valores dos erros produzidos pela comparação entre as estimativas de umidade

\begin{tabular}{lcrrrrrr}
\hline Tensão $\left(\mathrm{cm} \mathrm{H}_{2} \mathrm{O}\right)$ & 20 & \multicolumn{1}{c}{60} & 100 & 330 & 1000 & 5000 & 15000 \\
\hline Erro médio (\%) & 4,65 & 4,61 & 4,44 & 4,11 & 4,01 & 4,21 & 4,42 \\
Erro máximo (\%) & 12,31 & 15,81 & 15,85 & 10,42 & 10,09 & 10,64 & 10,51 \\
Erro mínimo (\%) & 0,029 & 0,19 & 0,05 & 0,73 & 0,47 & 0,26 & 0,22 \\
\hline
\end{tabular}

que se aumenta a tensão, em que se constatam elevados valores dos mesmos, reforçando a análise a respeito da qualidade dos modelos.

Assim, com base nos modelos propostos diz-se que as características hidrológicas do solo, destacando-se aquelas direta ou indiretamente associadas à curva característica do solo, são determinadas pelos atributos físico-hídricos do solo, especialmente textura, matéria orgânica e densidade do solo. Salienta-se, entretanto, que existe uma forte relação entre a gênese do solo e seu comportamento hidrológico, uma vez que esta determina, juntamente com o intemperismo do material de origem, os atributos físicos e químicos do solo, direcionando suas características comportamentais no tocante à interação água-solo, o que sugere que o modelo proposto tenha uso limitado a solos com características semelhantes às do trabalho em questão.

\section{CONCLUSÕES}

1. Os modelos desenvolvidos apresentam boas características estatísticas, com coeficientes de determinação elevados e baixos erros de predição, especialmente para os parâmetros $\theta \mathrm{R}, \theta \mathrm{S}$ e $\theta \mathrm{CC}$.

2. O modelo para o parâmetro alfa $(\alpha)$ foi o que gerou menor coeficiente de determinação, no entanto, produziu precisão considerável, com erro médio aceitável para modelos dessa natureza.

3. Com a equação de van Genuchten ajustada com parâmetros estimados pelos modelos propostos, obtiveram-se estimativas de boa precisão para as umidades do solo associadas às tensões trabalhadas, demonstrando que as curvas características estarão próximas da realidade física, permitindo aplicações dos modelos para este Cambissolo, na região estudada, desde que obedecidas as condições em que o trabalho foi desenvolvido.

\section{LITERATURA CITADA}

Arruda, F.B.; Zullo JR, J.; Oliveira, J.B. Parâmetros de solo para o cálculo da água disponível com base na textura do solo. Revista Brasileira de Ciência do Solo, Campinas, v.11, n.1, p.11-15, 1987.

Brasil. Ministério das Minas e Energia. Departamento Nacional da Produção Mineral. Projeto RADAM-BRASIL. Folhas S.F. 23/24 Rio de Janeiro/Vitória. Rio de Janeiro: Geologia, geomorfologia, pedologia, vegetação, uso potencial da terra. 1983. Rio de Janeiro, 1983. p.780. Levantamento de recursos naturais, 32
Centurion, J.F.; Andrioli, I. Regime hídrico de alguns solos de Jaboticabal. Revista Brasileira de Ciência do Solo, Viçosa, v.24, n.4, p.701-709, 2000.

Dias Junior, M.S.; Bertoni, J.C; Bastos, A.R.R. Física do solo. Lavras, UFLA, 2000. 147p.

EMBRAPA - Empresa Brasileira de Pesquisa Agropecuária. Centro Nacional de Pesquisa de Solos. Manual de métodos de análise de solos. 2.ed. Rio de Janeiro: 1997. 212p.

Fabian, A.J.; Ottoni Filho, T.B. Determinação de capacidade de campo in situ ou através de equações de regressão. Pesquisa Agropecuária Brasileira, Brasília, v.35, n.5, p.10291036, 2000.

Ferreira, M.M.; Marcos, Z.Z. Estimativa da capacidade de campo de Latossolo Roxo distrófico e Regossolo através do ponto de inflexão da curva característica de umidade. Ciência e Prática, Lavras, v.7, p. 96-101, 1983.

Gee, G.W.; Bauder, J.W. Particle-size analysis. In: Methods of soil analysis. Madison: American Society of Agronomy, 1986. p.383-411.

Giarola, N.F.B.; Curi, N.; Siquiera, J.O.; Chagas, C.S.; Ferreira, M.M. Solos da região sob influência do reservatório da hidrelétrica de Itutinga/Camargos, MG: perspectiva ambiental. Belo Horizonte, UFLA/CEMIG, 1997. 101p.

Gupta, S.C.; Larson, W.E. Estimating soil water retention characteristics from particle size distribution, organic matter content, and bulk density. Water Resources Research, Washington, v. 20, p.1633-1635, 1979.

Hodnett, M.G.; Tomasella, J. Marked differences between van Genuchten soil water-retention parameters for temperature and tropical soils: A new water-retention pedo-transfer functions developed for tropical soils. Geoderma, Amsterdam, v.108, p.155-180, 2002.

Libardi, P.L. Dinâmica da água no solo. Piracicaba: [s.n], 1995. 497p.

Mello, C.R. de; Oliveira, G.C. de; Ferreira, D.F.; Lima, J.M. de. Predição da porosidade drenável e disponibilidade de água para Cambissolos da microrregião Campos das Vertentes, MG. Pesquisa Agropecuária Brasileira. Brasília, v. 37, n.9, p.1319-1324, 2002a.

Mello, C.R. de; Oliveira, G.C. de; Resck, D.V.S.; Lima, J.M.; Dias Junior, M.S. Estimativa da capacidade de campo baseada no ponto de inflexão da curva característica. Revista Ciência e Agrotecnologia. Lavras, v. 26, n.4, p.836-841, 2002 b.

Mualem, Y. A new model for predicting the hydraulic conductivity of unsaturated porous media, Water Resource Research, Washington, v. 12, p.513-522, 1976.

Oliveira, G.C. de. Cambissolos da microrregião Campos da Mantiqueira, MG: caracterização físico-hídrica e interpretação para manejo. Lavras: UFLA, 1993. 62p. Dissertação Mestrado

Resende, M.; Curi, N.; Rezende, S.B.; Correa, G.F. Pedologia: base para distinção de ambientes. Viçosa: NEPUT, 1999. 338p.

Sales, L.E.O.; Ferreira, M.M.; Oliveira, M.S.; Curi, N. Estimativa da velocidade de infiltração básica do solo. Pesquisa Agropecuária Brasileira, Brasília, v.34, n.11, p.2091-2095, 1999. 
Silveira, A.L. da; Louzada, J.A.; Beltrame, L. Infiltração e armazenamento no solo. In: Tucci, C.E.M. (Org.) Hidrologia: ciência e aplicação. 2.ed. Porto Alegre: ABRH/Editora UFRGS, 2001.p.335-372.

SAS - Statistical Analyses System. Language guide for personal computers. 6. ed. Cary: SAS Institute, 1985. 429p.

Tomasella, J.; Hodnett, M.G. Estimating unsaturated hydraulic conductivity of Brazilian soils using soil-water retention data. Soil Science, Baltimore, v.162, p.703-712, 1997.

Tomasella, J.; Hodnett, M.G.; Rossato, L. Pedotransfer functions for the estimation of soil water retention in Brazilian soils. Soil Science Society of America Journal. Madison, v. 63, p.327-338, 2000. van Genuchten, M.T. van. A closed form equation for predicting the hydraulic conductivity of unsaturated soils. Soil Science Society of America Journal, Madison, v.44, p.892-898, 1980.

Walkley, A.; Black, I.A. An examination of the Degtjareff method for determining soil organic matter and a proposed modification of the chromic acid titration method. Soil Science, Baltimore, v.37, p. 29-38, 1934.

Wösten, J.H.M.; Schuren, C.H.J.E.; Bouma, J.; Stein, A. Functional sensitivity analysis of four methods to generate soil hydraulic functions. Soil Science Society of America Journal, Madison, v.55, p.832-836, 1990.

Zhuang, J.; Jin, Y.; Miyazaki, T. Estimating water retention characteristics from soil particle-size distribution using a non-similar media concept. Soil Science, Baltimore, v.166, n.5, p.308-321, 2001. 